\title{
Meta-analysis of risk factors for Parkinson's disease dementia
}

\author{
Yaqian Xu, Jing Yang and Huifang Shang ${ }^{*}$
}

\begin{abstract}
Background: Parkinson's disease (PD) is a common heterogeneous neurodegenerative disorder in elder population. Parkinson's disease dementia (PDD) is one of the most common non-motor manifestations in PD patients. No comprehensive review has been conducted to assess risk factors for PDD.

Methods: A systemic search for studies on PDD risk factors was performed. Cohort and case-control studies that clearly defined PDD and presented relevant data were included. The data were analyzed to generate a pooled effect size and $95 \%$ confidence interval (Cl). Publication bias was assessed using the Egger's test and the Begg's test.
\end{abstract}

Results: A systematic search was conducted and yielded 5195 articles. After screening, 25 studies were included in the current analysis. Development of PDD was positively associated with age (odds ratio [OR] 1.07, 95 \% Cl 1.03-1.13), male (OR 1.33, 95 \% Cl 1.08-1.64), higher Unified Parkinson's Disease Rating Scale (UPDRS) part III scores (relative risk [RR] 1.04, $95 \% \mathrm{Cl}$ 1.01-1.07), hallucination (OR 2.47, $95 \% \mathrm{Cl}$ 1.36-4.47), REM sleep behavior disorder (RBD) (OR 8.38, $95 \% \mathrm{Cl}$ 3.87-18.08), smoking (ever vs. never) (RR 1.93, $95 \% \mathrm{Cl} 1.15-3.26$ ) and hypertension (OR 1.57, $95 \% \mathrm{Cl} 1.11-2.22$ ). An inverse association was found between education (RR $0.94,95 \% \mathrm{Cl} 0.91-0.98$ ) and PDD. Other reported factors, including age of onset, disease duration of PD, Hoehn and Yahr stage and diabetes mellitus were not significantly associated with PDD.

Conclusions: Advanced age, male, higher UPDRS III scores, hallucination, RBD, smoking and hypertension increase the risk of PDD, whereas higher education is a protective factor for PDD.

Keywords: Parkinson's disease, Dementia, Risk factors, Predictors

\section{Background}

Parkinson's disease (PD), a heterogeneous neurodegenerative disorder in elder population, is characterized by cardinal motor symptoms including bradykinesia, rigidity, tremor and postural instability [1]. Recently, increasing evidence shows that PD is a disease with many non-motor symptoms (NMS) including dementia, sleep disorders, mood disorders, urinary dysfunction, and olfactory disorders [2]. Among NMS, Parkinson disease dementia (PDD) is one of the most common symptoms with a mean prevalence of $31.3 \%$ in PD patients [3]. Among general population, PDD incidence rate is approximately 38.7 to 112.5 per 1000 person-year among several cohort studies conducted in different regions [3, 4]. It has been suggested

\footnotetext{
* Correspondence: hfshang2002@163.com

Department of Neurology, West China Hospital, Sichuan University, 610041 Chengdu, Sichuan, China
}

that PD patients who developed dementia tend to have increased health care burden, declined quality of life and increased mortality [5-7]. However, effective treatment for PDD is currently unclear [8]. Being able to predict PDD development accurately would provide opportunities for intervention as well as novel treatments and might pro-

Several demographic, motor and non-motor features have been identified as predictors for PDD. Advanced age is the most common risk factor for dementia and for later diagnosis of PDD in PD patients [10]. More advanced disease stage as well as specific Parkinson subtype, the akinetic-rigid subtype, was found to be associated with increased risk for PDD, whereas the evaluation scales are not coherent [11, 12]. Some studies suggested that REM sleep behavior disorder (RBD), hallucination, mood disorders and olfactory dysfunction are strong predictors for 
PDD, but the results were not consistent across studies [13-15]. Up to date, no comprehensive meta-analysis on clinical risk factors for PDD has been conducted. A 2014 review on the predictors of PDD by Moore et al. summarized major study results on different risk factors, including clinical predictors, biological predictors, neuroimaging predictors and genetic predictors [9]. In that previous review, the authors presented all possible influences of those factors on PDD, but did not provide quantitative evaluation of the predictors. In order to quantitatively evaluate the effects of different factors on PDD, we conducted this systematic review and meta-analysis via an extensive search of observational studies and a meta-analysis on multiple factors.

\section{Methods}

\section{Search strategy}

We conducted the search according to the Preferred Reporting Items for Systematic Review and Meta-analysis (PRISMA 2009) guideline. We searched MEDLINE and EMBASE database for studies reporting predictors for later diagnosis of PDD. No language restrictions were used. The keywords we selected were: "Parkinson Disease" AND "Dementia" AND "Risk" OR "Predict" OR "Age" OR "Age of Onset" OR "Education" OR "Family history" OR "Hallucination" OR "Sleep Disorders" OR "Constipation" OR "Olfactory Disorders" OR "Color Vision" OR "Depression" OR "Anxiety" OR "Mood Disorders" OR "Erectile Dysfunction" OR "Urinary Dysfunction" OR "Hypertension" OR "Coronary Artery Disease" OR "Head Injury" OR "Diabetes Mellitus" OR "Smoking" OR "Alcohols" OR "Coffee" OR "Pesticides". We also hand searched the reference lists of relevant reviews and articles with required data for missed references. The final search was carried out on December 1, 2015.

\section{Inclusion criteria}

We included articles that met the predefined criteria: 1) cohort or case-control studies assessed at least one risk factor preceding a later diagnosis of PDD; 2) compared PDD patients with PD patients who did not develop dementia; 3 ) clearly stated diagnostic criteria for PD and PDD, and carried out by an experienced clinician; 4) reported odds ratio (OR), relative risk (RR) or equivalent values representing risks of developing dementia or case-control studies with cases defined as diagnosed PDD; and 5) reported data that could be easily obtained via questionnaires.

\section{Exclusion criteria}

Reviews, editorials, case reports, commentaries, letters that reported no new data, meta-analysis, handouts, and abstracts were excluded from the study. We excluded studies that: 1) reported on treatment or management of
PDD; 2) reported a diseases other than PDD or PD; 3) studied only young onset PD; 4 ) did not use a PD nondemented group as comparable group to PDD group or did not provide adequate data on the comparable group; 5) were twin studies; 6 ) reported one predictor repeatedly in one study population (if $>1$ paper reported on one study population, we chose the larger one, and where population is equal, we chose the most recent one); 7) reported on predictors that were not easily available in most clinical settings (i.e. questionnaires designed for a certain population or country); 8) reported only uncommon genetic risk factors; 9) reported measures other than OR, RR or equivalent values, or from which an OR could not be calculated. Two authors (X.Y. and J.Y.) independently evaluated the eligibility of all studies, and if there was disagreement between authors, the articles were further evaluated by a third author (H.S.) and discussed in detail until an agreement has been reached.

\section{Data extraction and quality assessment}

Study characteristics, PDD diagnostic criteria, a risk estimate of the main study finding, and secondary findings were extracted using a unified form. We did not include studies that reported dementia before or within one year to the onset of PD, since these cases did not fulfill the diagnostic criteria for PDD and were more likely cases of dementia with Lewy bodies (DLB). If studies did not report OR, RR or equivalent measures, raw data were screened to determine whether ORs could be calculated. When the studies reported both the crude OR/RRs and the adjusted OR/RRs, the adjusted figures were extracted. We calculated a quality score to assess the quality of the studies according to the Newcastle-Ottawa Scale (NOS). Length of time that any predictor precedes the diagnosis of PDD was not analyzed in the study due to inconsistent reporting.

\section{Statistical analysis}

We combined the reported risks first separately for case-control and cohort studies, and second for all studies together, in cases where two or more studies reported on the same factor. The data were analyzed to generate a pooled effect size and $95 \%$ confidence interval (CI). We examined the heterogeneity across studies using the $\mathrm{I}^{2}$ statistic $[16,17]$. Where statistically significant heterogeneity was found $(p<0.05)$, we used randomized effects model to combine results. We assessed publication bias using the Egger's test and the Begg's test, and constructed funnel plot in order to visualize any possible asymmetry [18]. Two-tailed $P$ values less than 0.05 were considered statistically significant. All analyses were performed using Stata version 12.0 (StataCorp, College Station, TX). 


\section{Results}

The electronic search yielded 5195 articles, all of which were reviewed by titles and abstracts. Full text of 278 articles were reviewed, of which 23 articles met the inclusion criteria. We also hand searched the references of the articles, 2 of which were included into the analysis. Finally, a total of 25 articles were included in the metaanalysis. Full details of the studies included were provided (Additional files 1 and 2). The selection process is shown in a flow diagram (Fig. 1).

We found age (OR 1.07, $95 \%$ CI 1.03-1.13), gender (Male) (OR 1.33, 95 \% CI 1.08-1.64) and hypertension (OR 1.57, $95 \%$ CI 1.11-2.22), to be significantly associated with later diagnosis of PDD (Fig. 2), whereas age of onset (AOO) (RR 1.03, $95 \%$ CI 0.97-1.09), disease duration of PD (RR 1.00, $95 \%$ CI 0.96-1.03), and type 2 diabetes mellitus (OR 1.16, $95 \%$ CI 0.58-2.42) were not associated with risk of PDD (Fig. 3). Although education was only reported in three cohort studies, it was the only factor we found to decrease PDD risk (RR 0.94, 95 \% CI 0.91-0.98) (Fig. 3). Lifestyle related risk factors were poorly reported. Previous and current smokers were reported to have increased risk of developing PDD (RR 1.88, $95 \% \mathrm{CI}$ 1.06-3.34) comparing with non-smoking PD population. However, alcohol consumption (RR 1.1, 95 \% CI 0.6-2.2) and coffee consumption (RR 0.9, $95 \%$ CI 0.5-1.8), reported in one study population, was not a significant risk factor for PDD [19].
Two general scales evaluating PD patient's motor features were included in this study. Our result showed that higher score in Unified Parkinson's Disease Rating Scale (UPDRS) part III (RR 1.04, 95 \% CI 1.01-1.07) increased the risk of PDD, but higher Hoehn and Yahr stage (RR $1.24,95 \%$ CI 0.92-1.66) was not associated with the development PDD. Studies analyzing the association between PD motor subtypes and PDD were not included in the present meta-analysis because of limited data. Two common non-motor symptoms of PD patients, hallucination (OR 2.47, 95 \% CI 1.36-4.47) and RBD (OR 8.38, 95 \% CI 3.87-18.08), were both strong predictors of PDD. Single studies also reported positive association between PDD and family history of dementia (first-degree relatives), urinary dysfunction, impaired color vision or orthostatic blood pressure drop and no significant association between family history of PD (first-degree relatives), exposure to pesticides, occupational exposure to chemicals and PDD $[13,15,20]$. Details of factors not included in the meta-analysis were provided (Additional file 3).

\section{Assessment of publication bias}

The funnel plot for each included factors were individually examined visually. The shape was presented essentially symmetrical in age, AOO, gender, smoking, disease duration, education, UPDRS III, hallucination, RBD, hypertension, diabetes mellitus and Hoehn and Yahr stage, which was proved by Begg's and Egger's test.

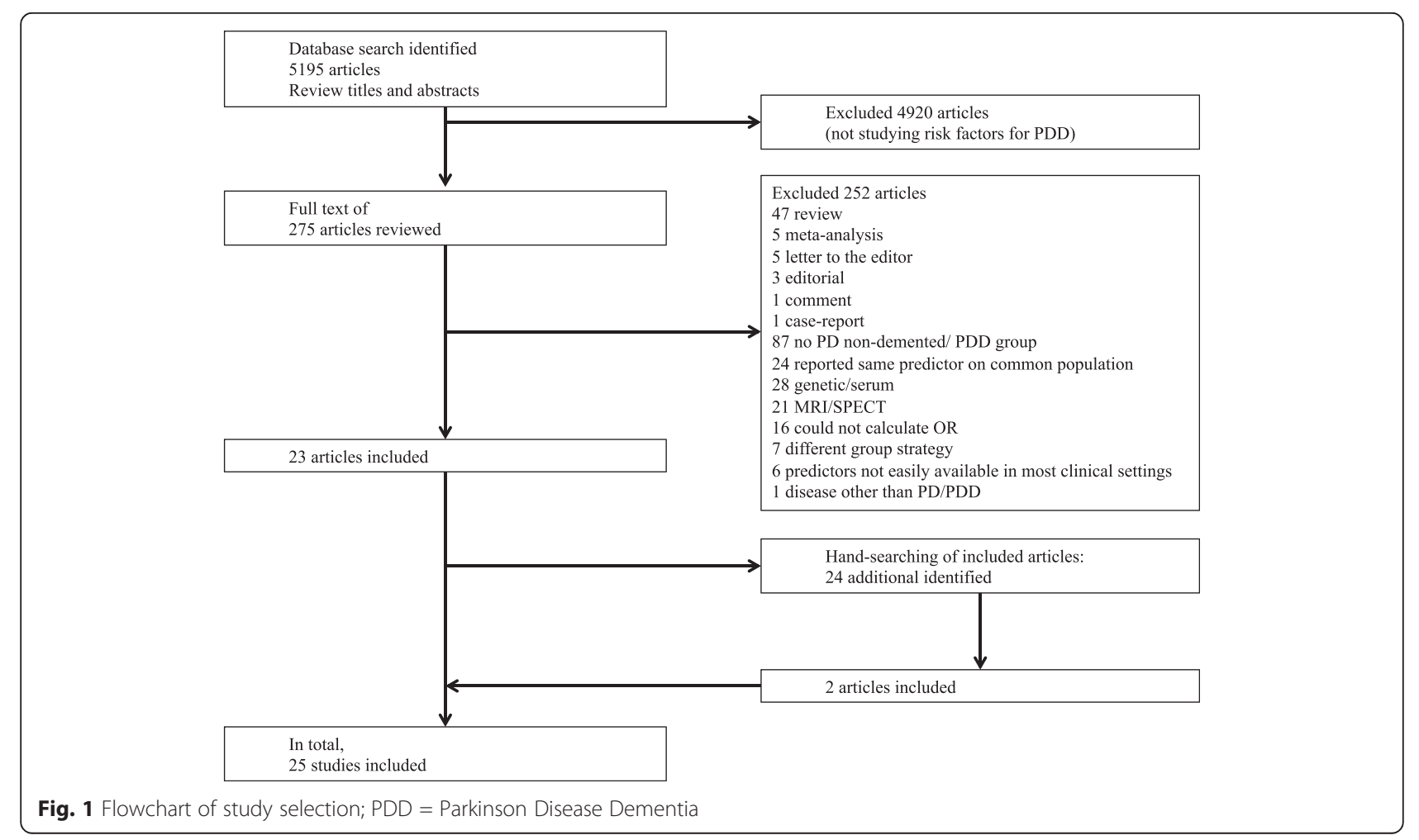




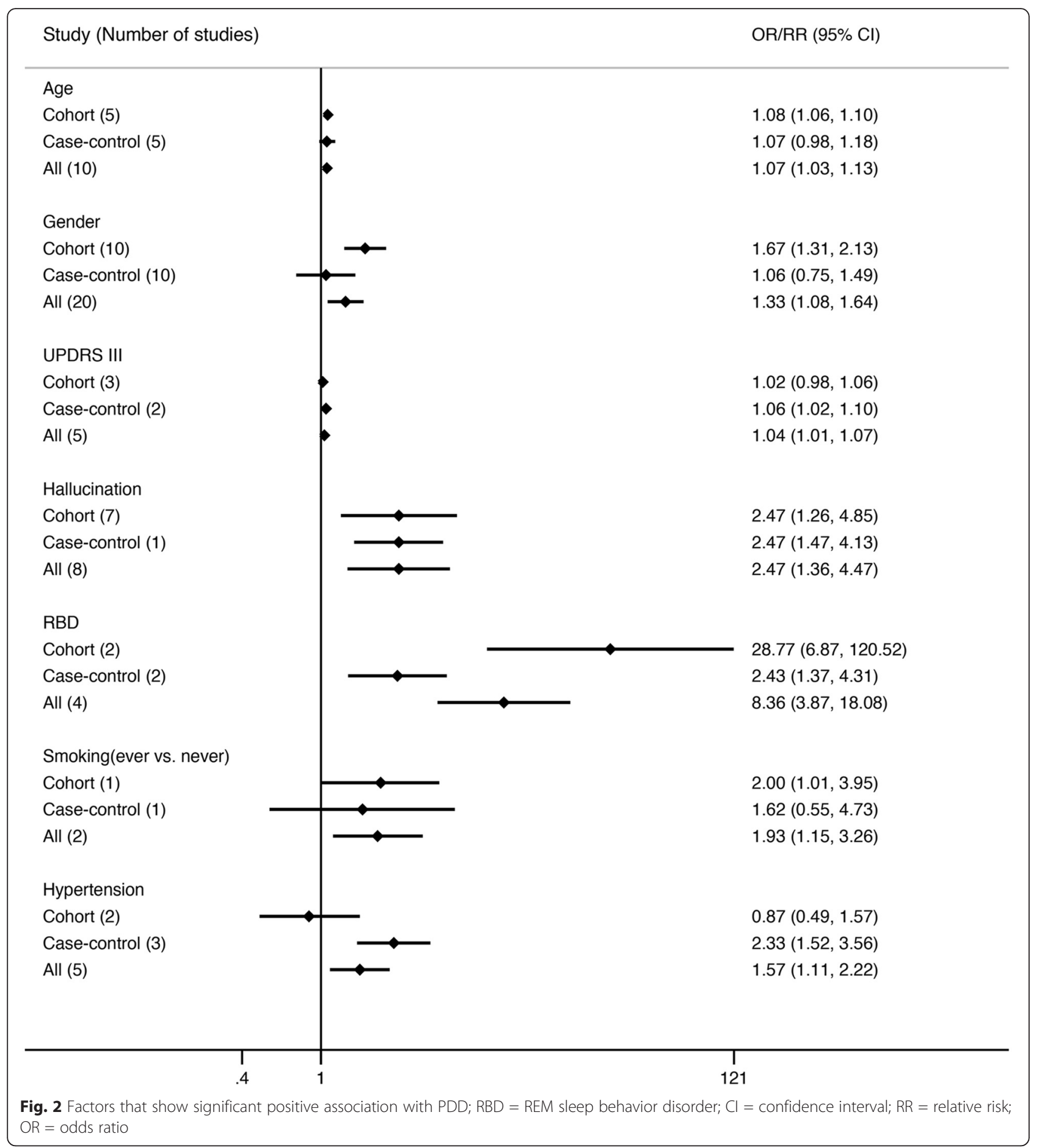

\section{Discussion}

This study identified 12 individual predictors that have potential value in screening for PDD. The identified risk factors include demographic characteristics, lifestyle factors, non-motor features of PD, and widely accepted scales evaluating PD. Some of the factors may present pathogenic importance while others could represent the relationship between symptoms and cognitive decline.
Together, these factors tend to be markers preceding diagnosis of PDD in PD patients. Of the identified factors, 7 factors were significant predictors for subsequent diagnosis of PDD, the understanding of which may contribute to higher quality of care and improve quality of life in PD patients.

Age, $\mathrm{AOO}$ and disease duration were all common risk factors for PDD. However, since these three factors are 


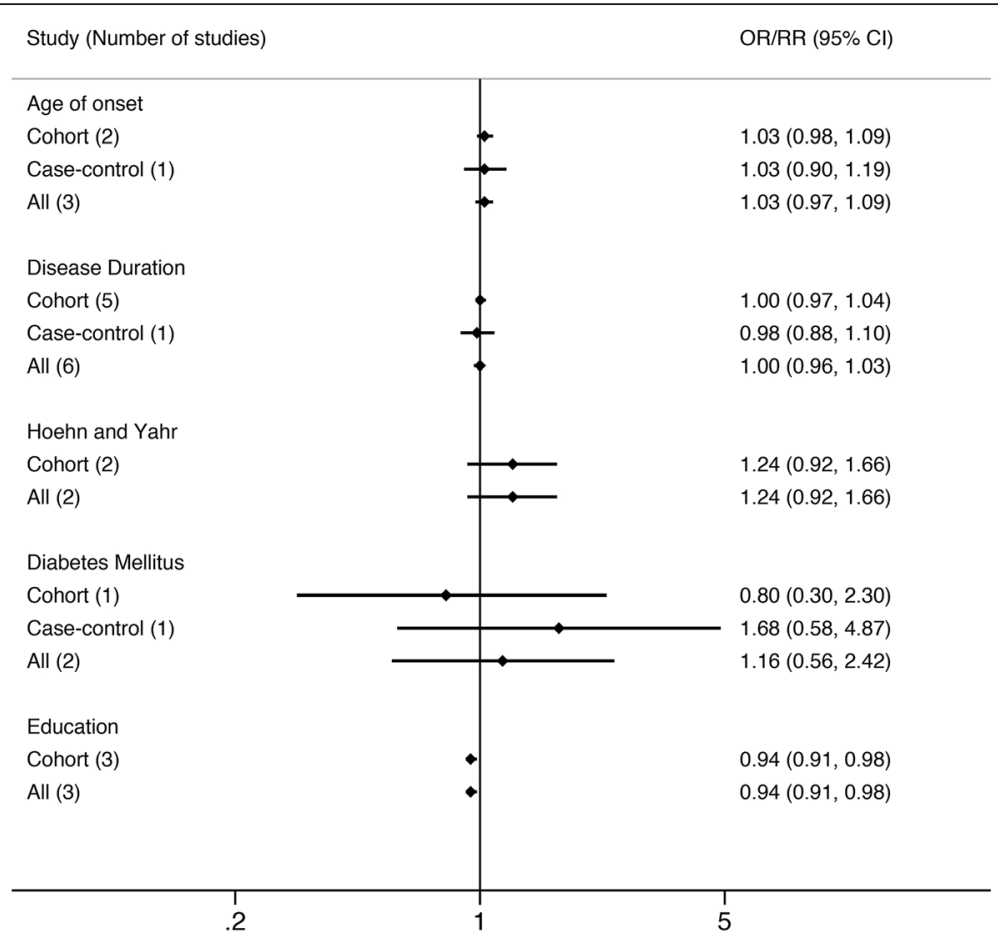

Fig. 3 Factor that shows significant negative association with PDD and factors that show no significant association with PDD; $\mathrm{Cl}=$ confidence interval; $\mathrm{RR}=$ relative risk; $\mathrm{OR}=$ odds ratio

interdependent, their individual effect on PDD is under debate. One study comparing early and late onset PD patients suggested that late onset PD group presents with more severe impaired sensory abilities, sleep disorders and dementia [21]. Another study adjusted cofounding found that among the three factors, only age remained an independent risk factor for PDD [22]. Similarly, we found that older age had a significant influence on later diagnosis of PDD, while AOO and disease duration was not associated with PDD. Our study suggested that advanced age as a risk factor for PDD may be independent from PD related time factors, like AOO or disease duration. Many neuropathological studies have provided evidences on the effect of aging. Recent studies with $\alpha$-synuclein immunostaining found a strong association between the age-related increase of Lewy bodies in cortical areas and the development of PDD [23, 24]. Another study found that cortical amyloid- $\beta$ deposition and aging together might be associated with PDD [25]. Few other studies revealed that MAPT genotype, related with tau transcription, has a strong influence on the risk of PDD [26, 27]. Goris et al. found that, among MAPT haplotypes, PD patients with $\mathrm{H} 1$ homozygotes had an increased rate of cognitive decline, which was dependent on age $[28,29]$. These age-related pathological processes together may increase the risk of PDD with aging.

We also found male to be a risk factor for PDD. However, some studies suggested that there might be no relationship between gender and PDD if potential confounding factors, including age, history of dementia, smoking and number of siblings, were adjusted [20, 22]. In the present meta-analysis, all data included for the factor gender were unadjusted data. Therefore, our study illustrated that male, before adjustment, is a positive predictor for PDD, but provided no evidence on the results after confounding factors were adjusted. The only protective factor we found in the current meta-analysis is higher education. Similar with our results, one study found that when compared with elementary school level, PDD patients with university level education were at lower risk of developing dementia [30]. One possible explanation is that education might modify the risk of cognitive decline by greater functional brain reserve in PD patients [31]. However, in a recent systematic review looking into education and dementia in Alzheimer's disease $(\mathrm{AD})$, the authors suggested that although lower education is associated with greater risk of dementia, the findings varied by region, age, gender and ethnicity [32]. The association between education and dementia in PD also need further studying with confounding adjusted.

A recent meta-analysis on risk factors for PD suggested that smoking, alcohol and coffee consumption decreases the risk of PD [33]. In the current meta-analysis we found that a history of smoking increased the risk of dementia in PD by almost two fold. On the other hand, alcohol and coffee consumption has been reported to have no significant 
association with PDD [19]. Smoking, different from alcohol and coffee consumption, stand as an independent risk factor for PDD. One possible mechanism is related with vascular factors. We found that hypertension was associated with PDD while diabetes was not significantly associated with PDD. Because smoking is also a risk factor for hypertension and that hypertension is related with ADtype pathologies in dementia, it is possible that smoking affect dementia in PD via vascular route [34, 35]. However, after adjustment for the possibility of confounding vascular factors in one included study, smoking still exists as an increased risk for PDD [19]. Biologically, greater depletion of cholinergic cells in the nucleus basalis of Meynert has been observed in PDD [36], yet the up-regulation of central nicotine acetylcholine receptors by nicotine contrasts the mechanism [37, 38]. Another published meta-analysis found that smoking was also a cause of cognitive decline in $\mathrm{AD}$ patients as well as in patients with other dementia, in which the authors suggested that non-smokers have lower inflammation or oxidative stress that may lead to a reduction in cognitive decline $[39,40]$.

We found that higher UPDRS III score was positively related with later diagnosis of PDD, whereas Hoehn and Yahr stage was not significantly associated with PDD. This result suggests that severe motor dysfunction is associated with PDD risk, and that the risk is more likely associated with individual motor dysfunctions. In a study that discovered no significant association between UPDRS III and PDD, researchers found that within the UPDRS III section, gait dysfunction was strongly associated with eventual development of dementia [13]. Gait dysfunction is important for the classification of the postural instability and gait difficulties (PIGD) subtype, and several longitudinal studies have discovered that $25 \%$ to $64.9 \%$ of PIGD PD patients would be diagnosed with PDD by the end of follow-up $[11,12]$. Also, researchers have found that increased loss of cholinergic nuclei may relate with both cognitive decline and motor features including rigidity, gait, and balance $[12,41]$. However, only two studies were evaluated regarding Hoehn and Yahr stage, the result should be interpreted with caution.

We discovered RBD and hallucination are strongly associated with later diagnosis of PDD. Several studies suggested that patients with RBD had a higher rate of MCI at baseline and a shorter duration towards diagnosis of dementia [42-44]. Cholinergic deficit due to degeneration of ascending pathway that took place in both RBD and dementia with Lewy bodies might be the cause [45]. Visual hallucinations were also discovered to have positive association with cognitive impairment in early PD [46]. In terms of mechanism, pathological studies in PD indicated that visual hallucination might share common limbic pathology with cognitive decline and dementia [14]. In functional MRI assessment, preceding image recognition, patients with visual hallucinations have reduced activation in ventral/lateral visual associated cortices [47]. However, a reverse relation was found in previous study suggesting that cognitive impairment at baseline precedes later development of hallucination [48]. Both RBD and hallucination were also associated with $\mathrm{MCI}$, an important predictor for PDD $[49,50]$, in early Parkinson disease $[42,46]$. None of the studies included in our meta-analysis adjusted RBD or hallucination for baseline cognitive impairment, therefore the causal relation is unclear from the present study.

\section{Limitations}

We only selected factors that were easily obtained in primary care environment. Variables that used less common inventories, like color vision and olfactory dysfunction, were not included in the analysis. Also, genetic tests have been excluded from the analysis for similar reasons. Mild cognitive impairment, an important risk factor of incidence dementia in PD $[50,51]$, was not included in the meta-analysis because most studies on mild cognitive impairment used a different group strategy by separating the participants into PD-normal cognition, PD-mild cognitive impairment and PDD group, which differs from most of other PDD risk factor studies. Motor subtypes were not analyzed in this meta-analysis also because of differences in group strategy.

Not all studies adjusted risk factors for confounders, and those adjusted were mostly adjusted for different confounders. We included both factors unadjusted and factors adjusted where possible, which may have increased the degree of significance for some risk factors. Few factors were reported in studies to be positively associated or not associated with later diagnosis of PDD but without specified OR or RR to be extracted. Though we have calculated OR or RR where possible, there may still be data neglected.

Statistically significant heterogeneity was found in 8 of the meta-analyses performed in our study. Two risk factors, age and AOO, were found to have high heterogeneity $\left(\mathrm{I}^{2}>75 \%\right)$. Gender, education, hallucination, UPDRS III, RBD and hypertension were found to have moderate heterogeneity $\left(50 \%<\mathrm{I}^{2}<75 \%\right)$. The presence of heterogeneity was as expected because of the differences in the characteristics of studies, the length of follow-up, study population scale, population characteristics, diagnostic criteria used and whether factors were crude or adjusted. The follow-up period in the cohort studies and the diagnostic criteria for PDD in both cohort and case-control studies varied, we did not adjust these factors in this meta-analysis due to limited number of included studies. Thus, the results of this analysis should be interpreted cautiously, especially for those factors that were reported in less than three studies and that were with high heterogeneities. 
In the present meta-analysis, we included studies with NOS score higher than 5 , which ensured study quality. Also, we performed sub-group analysis by different study design in order to minimize heterogeneity. We combined analytical results according to the heterogeneity analysis, and did not find significant publication bias. Therefore, our results were considered to be robust.

\section{Conclusions}

This is the first systematic review and meta-analysis on widely evaluated risk factors of PDD. This study found advanced age, male, high UPDRS III scores, presence of hallucination, presence of RBD, ever smoking and history of hypertension are positive predictors to later diagnosis of PDD, whereas education is a protective factor of PDD. This study laid the foundation to future comparative assessment on risk factors for PDD, and lead to a better understanding of PDD risks.

\section{Additional files}

Additional file 1: Details of studies included in the meta-analysis. This file included details of all studies included in this meta-analysis, categorized by risk factors. Details including first author, year of publication, country, study design, number of participants and NOS scores. (DOCX $119 \mathrm{~kb}$ )

Additional file 2: Details of the study results. This file provided detailed results of meta-analysis of each risk factor. (DOCX $159 \mathrm{~kb})$

Additional file 3: Details of factors not included in the meta-analysis. This file provided data of risk factors for Parkinson's disease dementia that were mentioned in literature, but were not included in the meta-analysis due to limited number of studies or differences in study design. (DOCX $93 \mathrm{~kb}$ )

\section{Abbreviations}

$A D$, Alzheimer's disease; $A O O$, age of onset; $C l$, confidence interval; $\mathrm{DLB}$ dementia with lewy bodies; NOS, Newcastle-Ottawa scale; OR, odds ratio; PD, Parkinson's disease; PDD, Parkinson's disease dementia; PIGD, postural instability and gait difficulties; PRISMA, preferred reporting items for systematic review and meta-analysis; $\mathrm{RBD}$, REM sleep behavior disorder; $\mathrm{RR}$, relative risk; UPDRS, Unified Parkinson's Disease Rating Scale.

\section{Acknowledgement}

We would like to thank all authors of the original research studies included in this meta-analysis. We thank Ms. Cong Li for statistical assistance and thank Ms. Hong Xie for proofreading of this meta-analysis.

\section{Funding}

No funding considering this study.

\section{Availability of data and materials}

The datasets supporting the conclusions of this article are included in the article and its additional files.

\section{Authors' contributions}

$Y X$ and HS conceived and designed the study. $Y X, J Y$ and HS reviewed the literature. $Y X$ undertook the statistical analysis. $Y X$ and HS wrote the manuscript. $Y X, J Y$ and HS contribute in discussions and reviewed the manuscript. All authors read and approved the final manuscript.

\section{Authors' information}

Huifang Shang, corresponding author, professor and vice director of Department of Neurology, West China Hospital of Sichuan University, Chengdu, 610041, China.

\section{Competing Interests}

The authors declare that they have no competing interests.

Consent for publication

Not applicable.

Ethics approval and consent to participate

Not applicable.

Received: 7 March 2016 Accepted: 25 May 2016

Published online: 01 June 2016

References

1. de Lau LM, Giesbergen PC, de Rijk MC, Hofman A, Koudstaal PJ, Breteler MM. Incidence of parkinsonism and Parkinson disease in a general population: the Rotterdam Study. Neurology. 2004;63:1240-4.

2. Schapira AH. The measurement and importance of non-motor symptoms in Parkinson disease. Eur J Neurol. 2015:22:2-3.

3. Aarsland D, Kurz MW. The epidemiology of dementia associated with Parkinson's disease. Brain Pathol (Zurich, Switzerland). 2010;20:633-9.

4. Hobson P, Meara J. Risk and incidence of dementia in a cohort of older subjects with Parkinson's disease in the United Kingdom. Mov Disord. 2004; 19:1043-9.

5. Hely MA, Reid WG, Adena MA, Halliday GM, Morris JG. The Sydney multicenter study of Parkinson's disease: the inevitability of dementia at 20 years. Mov Disord. 2008;23:837-44.

6. Leroi I, McDonald K, Pantula H, Harbishettar V. Cognitive impairment in Parkinson disease: impact on quality of life, disability, and caregiver burden. J Geriatr Psychiatry Neurol. 2012;25:208-14.

7. Emre M, Aarsland D, Brown R, Burn DJ, Duyckaerts C, Mizuno Y, et al. Clinical diagnostic criteria for dementia associated with Parkinson's disease. Mov Disord. 2007:22:1689-707. quiz 837.

8. Marder K, Tang MX, Alfaro B, Mejia H, Cote L, Jacobs D, et al. Postmenopausal estrogen use and Parkinson's disease with and without dementia. Neurology. 1998;50:1141-3.

9. Moore SF, Barker RA. Predictors of Parkinson's disease dementia: towards targeted therapies for a heterogeneous disease. Parkinsonism Relat Disord. 2014;20 Suppl 1:S104-7.

10. Aarsland D, Kvaloy JT, Andersen K, Larsen JP, Tang MX, Lolk A, et al. The effect of age of onset of PD on risk of dementia. J Neurol. 2007;254:38-45.

11. Alves G, Larsen JP, Emre M, Wentzel-Larsen T, Aarsland D. Changes in motor subtype and risk for incident dementia in Parkinson's disease. Mov Disord. 2006:21:1123-30.

12. Burn DJ, Rowan EN, Allan LM, Molloy S, O'Brien JT, McKeith IG. Motor subtype and cognitive decline in Parkinson's disease, Parkinson's disease with dementia, and dementia with Lewy bodies. J Neurol Neurosurg Psychiatry. 2006;77:585-9.

13. Anang JB, Gagnon JF, Bertrand JA, Romenets SR, Latreille V, Panisset M, et al. Predictors of dementia in Parkinson disease: A prospective cohort study. Neurology. 2014;83:1253-60

14. Zhu K, van Hilten JJ, Putter H, Marinus J. Risk factors for hallucinations in Parkinson's disease: results from a large prospective cohort study. Mov Disord. 2013;28:755-62.

15. Baba T, Kikuchi A, Hirayama K, Nishio Y, Hosokai Y, Kanno S, et al. Severe olfactory dysfunction is a prodromal symptom of dementia associated with Parkinson's disease: a 3 year longitudinal study. Brain. 2012;135:161-9.

16. Higgins JP, Thompson SG, Deeks JJ, Altman DG. Measuring inconsistency in meta-analyses. BMJ (Clinical research ed). 2003:327:557-60.

17. DerSimonian R, Laird N. Meta-analysis in clinical trials. Control Clin Trials. 1986;7:177-88.

18. Egger M, Smith GD, Phillips AN. Meta-analysis: principles and procedures. BMJ (Clinical research ed). 1997;315:1533-7.

19. Levy G, Tang MX, Cote LJ, Louis ED, Alfaro B, Mejia H, et al. Do risk factors for Alzheimer's disease predict dementia in Parkinson's disease? An exploratory study. Mov Disord. 2002;17:250-7.

20. Marder K, Flood P, Cote L, Mayeux R. A pilot study of risk factors for dementia in Parkinson's disease. Mov Disord. 1990:5:156-61.

21. Zhou MZ, Gan J, Wei YR, Ren XY, Chen W, Liu ZG. The association between non-motor symptoms in Parkinson's disease and age at onset. Clin Neurol Neurosurg. 2013;115:2103-7. 
22. Hughes TA, Ross HF, Musa S, Bhattacherjee $S$, Nathan RN, Mindham RH, et al. A 10-year study of the incidence of and factors predicting dementia in Parkinson's disease. Neurology. 2000;54:1596-602.

23. Mattila PM, Rinne JO, Helenius H, Dickson DW, Roytta M. Alpha-synucleinimmunoreactive cortical Lewy bodies are associated with cognitive impairment in Parkinson's disease. Acta Neuropathol. 2000;100:285-90.

24. Hurtig HI, Trojanowski JQ, Galvin J, Ewbank D, Schmidt ML, Lee VM, et al. Alpha-synuclein cortical Lewy bodies correlate with dementia in Parkinson's disease. Neurology. 2000;54:1916-21.

25. Compta Y, Parkkinen L, O'Sullivan SS, Vandrovcova J, Holton JL, Collins C, et al. Lewy- and Alzheimer-type pathologies in Parkinson's disease dementia: which is more important? Brain. 2011;134:1493-505.

26. Seto-Salvia N, Clarimon J, Pagonabarraga J, Pascual-Sedano B, Campolongo A, Combarros O, et al. Dementia risk in Parkinson disease: disentangling the role of MAPT haplotypes. Arch Neurol. 2011:68:359-64.

27. Williams-Gray CH, Mason SL, Evans JR, Foltynie T, Brayne C, Robbins TW, et al. The CamPalGN study of Parkinson's disease: 10-year outlook in an incident population-based cohort. J Neurol Neurosurg Psychiatry. 2013;84: 1258-64.

28. Winder-Rhodes SE, Hampshire A, Rowe JB, Peelle JE, Robbins TW, Owen AM, et al. Association between MAPT haplotype and memory function in patients with Parkinson's disease and healthy aging individuals. Neurobiol Aging. 2015;36:1519-28.

29. Goris A, Williams-Gray CH, Clark GR, Foltynie T, Lewis SJ, Brown J, et al. Tau and alpha-synuclein in susceptibility to, and dementia in. Parkinson's Disease Ann Neurol. 2007;62:145-53.

30. Zoccolella S, dell'Aquila C, Abruzzese G, Antonini A, Bonuccelli U, Canesi M, et al. Hyperhomocysteinemia in levodopa-treated patients with Parkinson's disease dementia. Mov Disord. 2009;24:1028-33.

31. Glatt SL, Hubble JP, Lyons K, Paolo A, Troster Al, Hassanein RE, et al. Risk factors for dementia in Parkinson's disease: effect of education. Neuroepidemiology. 1996;15:20-5.

32. Sharp ES, Gatz M. Relationship between education and dementia: an updated systematic review. Alzheimer Dis Assoc Disord. 2011;25:289-304.

33. Noyce AJ, Bestwick JP, Silveira-Moriyama L, Hawkes CH, Giovannoni G, Lees AJ, et al. Meta-analysis of early nonmotor features and risk factors for Parkinson disease. Ann Neurol. 2012;72:893-901.

34. Virdis A, Giannarelli C, Neves MF, Taddei S, Ghiadoni L. Cigarette smoking and hypertension. Curr Pharm Des. 2010;16:2518-25.

35. Iadecola C. Hypertension and dementia. Hypertension. 2014;64:3-5.

36. Jellinger KA. Morphological substrates of dementia in parkinsonism. A critical update. J Neural Transm Suppl. 1997;51:57-82.

37. Grenhoff J, Svensson TH. Pharmacology of nicotine. Br J Addict. 1989;84 477-92.

38. Levin ED. Nicotinic systems and cognitive function. Psychopharmacology. 1992;108:417-31.

39. Anstey KJ, von Sanden C, Salim A, O'Kearney R. Smoking as a risk factor for dementia and cognitive decline: a meta-analysis of prospective studies. Am J Epidemiol. 2007;166:367-78.

40. Bruno RS, Traber MG. Vitamin E biokinetics, oxidative stress and cigarette smoking. Pathophysiology. 2006;13:143-9.

41. Bohnen NI, Kaufer DI, Ivanco LS, Lopresti B, Koeppe RA, Davis JG, et al. Cortical cholinergic function is more severely affected in parkinsonian dementia than in Alzheimer disease: an in vivo positron emission tomographic study. Arch Neurol. 2003:60:1745-8.

42. Postuma RB, Bertrand JA, Montplaisir J, Desjardins C, Vendette M, Rios Romenets S, et al. Rapid eye movement sleep behavior disorder and risk of dementia in Parkinson's disease: a prospective study. Mov Disord. 2012;27: 720-6.

43. Marion MH, Qurashi M, Marshall G, Foster O. Is REM sleep behaviour disorder (RBD) a risk factor of dementia in idiopathic Parkinson's disease? J Neurol. 2008:255:192-6.

44. Nomura T, Inoue Y, Kagimura T, Nakashima K. Clinical significance of REM sleep behavior disorder in Parkinson's disease. Sleep Med. 2013;14:131-5.

45. Emre M. Dementia in Parkinson's disease: cause and treatment. Curr Opin Neurol. 2004;17:399-404.

46. UC EY, McDermott MP, Marder KS, Anderson SW, Litvan I, Como PG, et al. Incidence of and risk factors for cognitive impairment in an early Parkinson disease clinical trial cohort. Neurology. 2009;73:1469-77.

47. Meppelink AM, de Jong BM, Renken R, Leenders KL, Cornelissen FW, van Laar T. Impaired visual processing preceding image recognition in Parkinson's disease patients with visual hallucinations. Brain. 2009; 132:2980-93.

48. van Rooden SM, Heiser WJ, Kok JN, Verbaan D, van Hilten JJ, Marinus J. The identification of Parkinson's disease subtypes using cluster analysis: a systematic review. Mov Disord. 2010;25:969-78.

49. Pedersen KF, Larsen JP, Tysnes OB, Alves G. Prognosis of mild cognitive impairment in early Parkinson disease: the Norwegian ParkWest study. JAMA Neurol. 2013;70:580-6.

50. Litvan I, Aarsland D, Adler CH, Goldman JG, Kulisevsky J, Mollenhauer B, et al. MDS Task Force on mild cognitive impairment in Parkinson's disease: critical review of PD-MCI. Mov Disord. 2011:26:1814-24.

51. Janvin CC, Larsen JP, Aarsland D, Hugdahl K. Subtypes of mild cognitive impairment in Parkinson's disease: progression to dementia. Mov Disord. 2006;21:1343-9.

\section{Submit your next manuscript to BioMed Central and we will help you at every step:}

- We accept pre-submission inquiries

- Our selector tool helps you to find the most relevant journal

- We provide round the clock customer support

- Convenient online submission

- Thorough peer review

- Inclusion in PubMed and all major indexing services

- Maximum visibility for your research

Submit your manuscript at www.biomedcentral.com/submit
) Biomed Central 